\title{
Unexpected coherence and conservation
}

\author{
Bernard Cazelles $^{1 *}$, Samuele Bottani ${ }^{2}$ and Lewi Stone ${ }^{3}$ \\ ${ }^{1}$ CNRS UMR 7625-Université Pierre et Marie Curie, 7 quai Saint Bernard, CC 237, 75252 Paris, France, and UFR de Biologie, \\ Université Paris 7-Denis Diderot \\ ${ }^{2}$ Modélisation Physique Interfaces Biologie, UFR de Physique, Université Paris 7-Denis Diderot, CC 7021, 75251 Paris, France \\ ${ }^{3}$ The Porter Super-Center for Ecological and Environmental Studies, and Department of Zoology, Tel Aviv University, Ramat Aviv, \\ Tel Aviv 69978, Israel
}

\begin{abstract}
The effects of migration in a network of patch populations, or metapopulation, are extremely important for predicting the possibility of extinctions both at a local and a global scale. Migration between patches synchronizes local populations and bestows upon them identical dynamics (coherent or synchronous oscillations), a feature that is understood to enhance the risk of global extinctions. This is one of the central theoretical arguments in the literature associated with conservation ecology. Here, rather than restricting ourselves to the study of coherent oscillations, we examine other types of synchronization phenomena that we consider to be equally important. Intermittent and out-of-phase synchronization are but two examples that force us to reinterpret some classical results of the metapopulation theory. In addition, we discuss how asynchronous processes (for example, random timing of dispersal) can paradoxically generate metapopulation synchronization, another non-intuitive result that cannot easily be explained by the standard theory.
\end{abstract}

Keywords: metapopulation dynamics; population synchrony; imperfect population synchrony; on-off intermittency; out-of-phase synchrony

\section{INTRODUCTION}

Ecosystems are spatially extended dynamical systems, and it is this spatial dimension that has profound implications for the persistence of all constituent populations. The effects of dispersal over the spatial landscape are important in this respect. If we consider a metapopulation (i.e. a set of patches or local patch populations), migration between patches might not only promote the persistence of local populations (Allen et al. 1993; Hassel et al. 1994), but it can also serve to synchronize them-a factor that increases the danger of global extinction. For example, if all local populations collectively synchronize to the same dangerously low level after a disturbance, this will tend to heighten the global extinction risk. However, if patch populations are asynchronous, any local patches that are perturbed to low numbers can more easily be 'rescued' from extinction by immigrants from safer and more populated neighbouring patches of relatively high population abundances (den Boer 1981). This is the well known 'rescue effect' often documented by ecologists (Gotelli 2001). As we shall see, the idea of synchrony and asynchrony in spatial populations becomes even more interesting when we extend our study to cases in which the dynamics of the populations are themselves complex or chaotic.

Recently Earn et al. (2000), when analysing the viability of a metapopulation, revisited general conditions under which coupled populations can or cannot undergo coherent synchronized oscillations. In their terminology, coherent oscillations are equivalent to the simplest possible form of synchronization, in which all local populations become identical and cannot be differentiated from one another in terms of abundance level. If

*Author for correspondence (bcazelle@snv.jussieu.fr). coherent, all local populations follow the same periodic or chaotic trajectory in time. Earn et al. (2000) generalized previous results from theoretical literature about synchronization of coupled map lattices (Kaneko 1990; Gade 1996; Ding \& Yang 1997; Dmitriev et al. 1997; Kozma 1998; Andreyev \& Dmitriev 1999; Gade \& Hu 1999) in an ecological context. Their results are of great interest for conservation biology, because they relate to the extinction likelihood of endangered populations and its dependence on the dispersal routes between patches within the metapopulation.

Earn et al. (2000) established a criterion for the stability of the synchronized coherent state that is related to only three key ecological parameters: the maximal growth rate of the population $r$, the eigenvalues of the dispersal matrix $\lambda$ (that depend on the size of the metapopulation $n$ ), and the fitness coefficient computed as the Lyapunov exponent of the net population growth $\mu$. They argued that coherent oscillations will never occur in the 'coherence impossibility region', defined by their criterion:

$\mathrm{e}^{\mu} \times|\lambda|>1$

This criterion is useful for the study of coherent oscillations, but identical coherent oscillations are only a single specific form of synchronization. In fact, synchronization is a far more general phenomenon (Rulkov et al. 1995; Rosenblum et al. 1996, 1997; Blasius et al. 1999; Blasius \& Stone 2000; Maistrenko et al. 2000) and has many subtle aspects that are equally worthy of consideration. Here, we show that synchronization is a complex phenomenon that can easily go unidentified, yet plays an important role even in the 'coherence impossibility region' of Earn et al. (2000). We will illustrate this point in the following, emphasizing that dynamics of metapopulations can be more unpredictable and more complex than expected. 

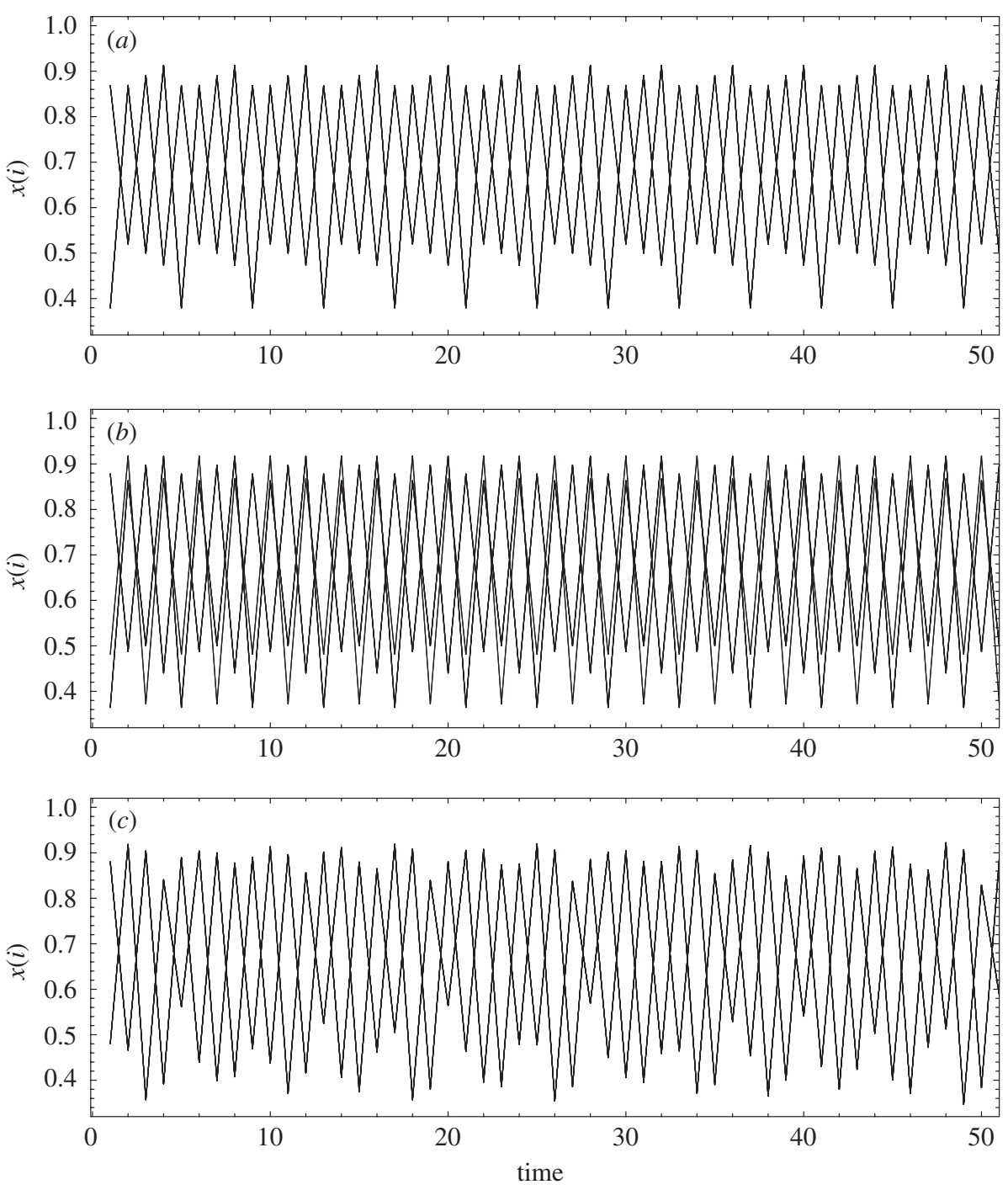

Figure 1. Clustering dynamics in the case of a logistic metapopulation of $n=10$ globally coupled populations. All the time iterations of the 10 populations are plotted on each graph. (a) Two clusters and period 4 dynamics; $(b)$ three clusters and period 4 dynamics; $(c)$ two clusters and complex (chaotic) dynamics. Parameters values are as follows: $(a) r=3.9, m=0.235$;

(b) $r=3.905, m=0.205$, and $(c) r=3.9, m=0.175$.

\section{THE MODEL}

Similar to a number of recent theoretical studies (Allen et al. 1993; Heino et al. 1997; Solé \& Gamarra 1998; Silva et al. 2000; Earn et al. 2000) we analyse a spatially structured discrete time metapopulation model often referred to as a 'coupled map lattice'

$x_{i}^{t+1}=\sum_{j=1}^{n} m_{i j} \times F\left(x_{j}^{t}\right) \quad i=1,2, \ldots, n$.

Here $x_{i}^{t}$ denotes the population density of the $i$ th patch at time $t$. The function $F\left(x_{i}\right)$ characterizes the birth-death processes of the local population $x_{i}$, and as is customary to fix ideas we make use of the logistic map

$F\left(x_{i}\right)=r \times x_{i} \times\left(1-x_{i}\right)$,

where $r$ defines the net growth rate of the local population. In the case when there is no migration between sites $\left(m_{i j}=0\right.$, $\left.m_{i i}=1\right)$, all $n$ populations are governed by the same map, i.e.

$x_{i}^{t+1}=F\left(x_{i}^{t}\right) \quad i=1,2, \ldots, n$.
The coefficients $m_{i j}$ in equation (2.1) may be written in the form of a dispersal matrix $M$ that quantifies the dispersal from patch $j$ to patch $i$ in the network of $n$ patches. For 'all-to-all' global coupling, the dispersal matrix $M$ is

$$
M=\left(\begin{array}{ccccc}
1-m & m /(n-1) & m /(n-1) & \cdots & m /(n-1) \\
m /(n-1) & 1-m & m /(n-1) & \cdots & m /(n-1) \\
m /(n-1) & m /(n-1) & 1-m & \cdots & m /(n-1) \\
\vdots & \vdots & \vdots & \ddots & \vdots \\
m /(n-1) & m /(n-1) & m /(n-1) & \cdots & 1-m
\end{array}\right)
$$

and for local coupling it is

$$
M=\left(\begin{array}{ccccc}
1-m & m / 2 & 0 & \cdots & m / 2 \\
m / 2 & 1-m & m / 2 & \cdots & 0 \\
0 & m / 2 & 1-m & \cdots & 0 \\
\vdots & \vdots & \vdots & \ddots & \vdots \\
m / 2 & 0 & 0 & \cdots & 1-m
\end{array}\right) .
$$


This simple set-up provides a convenient framework for examining how the dispersal matrix $M$ affects synchronization between the populations $x_{i}$. If all populations synchronize identically with $x_{i}=x_{j}$, for all $i, j$, then this is referred to as coherent oscillations. All populations in this case are identical and thus 'in phase' with one another to form a single coherent structure, and equation (2.1) collapses to equation (2.3). Many other synchronized solutions are possible. For example, different subsets of the populations might synchronize identically to form a cluster. Different clusters might have their own characteristic dynamics (e.g. periodic or chaotic) and some clusters might be 'out of phase' with others. Figure 1 illustrates this point and shows a configuration of two clusters (figure $1 a, c$ ) and three clusters in (figure $1 b$ ) with periodic (figure $1 a, b$ ) or chaotic (figure $1 c$ ) dynamics. A more thorough analysis of these complex behaviours can be found in Kaneko (1990) and Balmforth et al. (1999), for example.

\section{STABILITY AND SYNCHRONIZATION}

In the jargon of dynamical systems, perfect synchronization (i.e. 'coherent oscillations') defines an invariant manifold with $x_{i}=x_{j}$ for all $i, j$, so that all local populations are identical. The quantity behind the criterion of Earn et al. (2000, their eqn (2)) is based on the transverse Lyapunov exponent $\left(\mu_{\perp}\right)$ that determines the stability of this invariant manifold:

$\mu_{\perp}=\mu+\ln (|\lambda|)$,

with $\mu$ the Lyapunov exponent of the birth-death function in a single uncoupled patch (i.e. the quadratic logistic model (equation (2.2)) in our example) and $\lambda$ the subdominant eigenvalue of the dispersal matrix $M$.

As stressed by Earn et al. (2000), if $\mu_{\perp}>0$ the invariant synchronous manifold is unstable and perfect synchrony cannot occur; conversely, if $\mu_{\perp}<0$ the invariant manifold is stable and perfect synchrony can occur. In the case of the globally coupled version of metapopulation above, the subdominant eigenvalue $(\lambda)$ of the dispersal matrix is given by (Kaneko 1990; Ding \& Yang 1997; Kozma 1998)

$\lambda=\frac{n-1-n \times m}{n-1}$,

where $n$ is the number of patches and $m$ is the dispersal coefficient. The transverse Lyapunov exponent is thus

$\mu_{\perp}=\mu+\ln \left(\left|\frac{n-1-n \times m}{n-1}\right|\right)$.

For example, for $n=10$ globally coupled logistic maps with $r=4$ and $\mu=\ln (2)$, the 'coherence impossibility region' is defined by $m<m_{\mathrm{c}}=0.45$. Figure 2 makes clear the extent of the 'coherence impossibility region' over the relevant $(r, m)$ parameter space for these $n=10$ coupled populations in cases both with global and with local coupling.

It is important to note that the criteria established by Earn et al. (2000) are based on long-term average quantities (e.g. $\mu$ ). The finite time fluctuations of $\mu(t)$ do not guarantee areas on the attractor in the invariant subspace that are always locally transversally stable or unstable. In the case of complex dynamics (chaotic or stochastic),
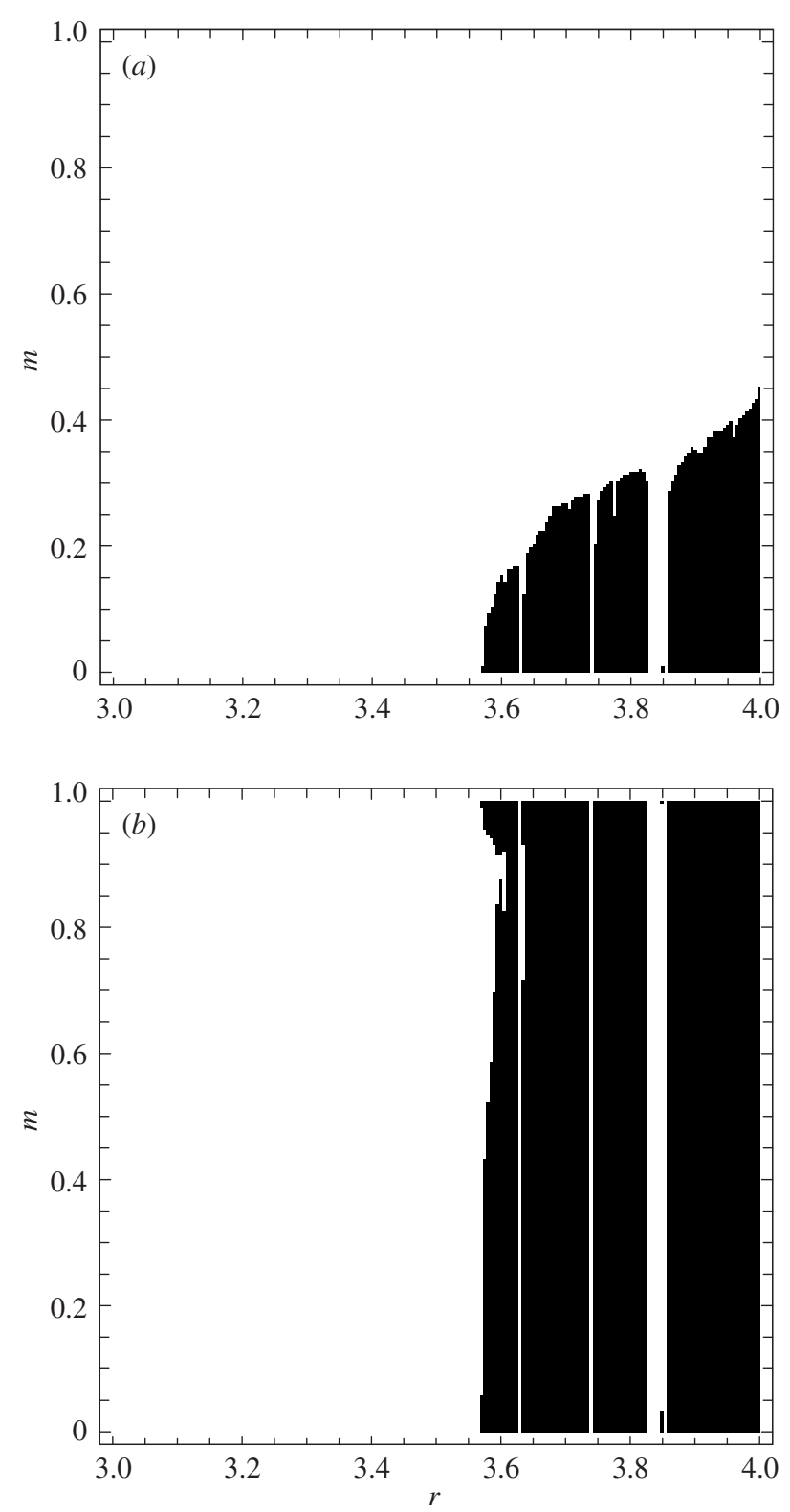

Figure 2. Coherence impossibility region (the black region), where $\mu_{\perp}>0$, in a logistic metapopulation of $n=10$ populations as a function of the net growth rate $r$ and the dispersal coefficient $m$, for global $(a)$ and local coupling $(b)$.

these quantities $\left(\mu, \mu_{\perp}\right)$ may fluctuate and their distributions may explain unexpected and unpredictable dynamics (e.g. riddled basins, on-off intermittency) (Ashwin et al. 1994, 1996; Lai et al. 1996). Hence, even when the invariant synchronous manifold is stable $\left(\mu_{\perp} \lesssim 0\right)$, there can still be initial conditions for which the transverse instantaneous Lyapunov exponent becomes positive and trajectories can move to another attractor. In this case, one may have riddled basins: depending on initial conditions we can observe perfect synchrony or non-coherent oscillations (Alexander et al. 1992). Furthermore, the riddled basins are fractal so that any improvement in the accuracy of initial conditions has little influence regarding which asymptotic attractor is reached by the trajectories (Alexander et al. 1992). Conversely, when this synchronous manifold is unstable $\left(\mu_{\perp} \gtrsim 0\right)$, it is still possible to observe synchronized dynamics, although 

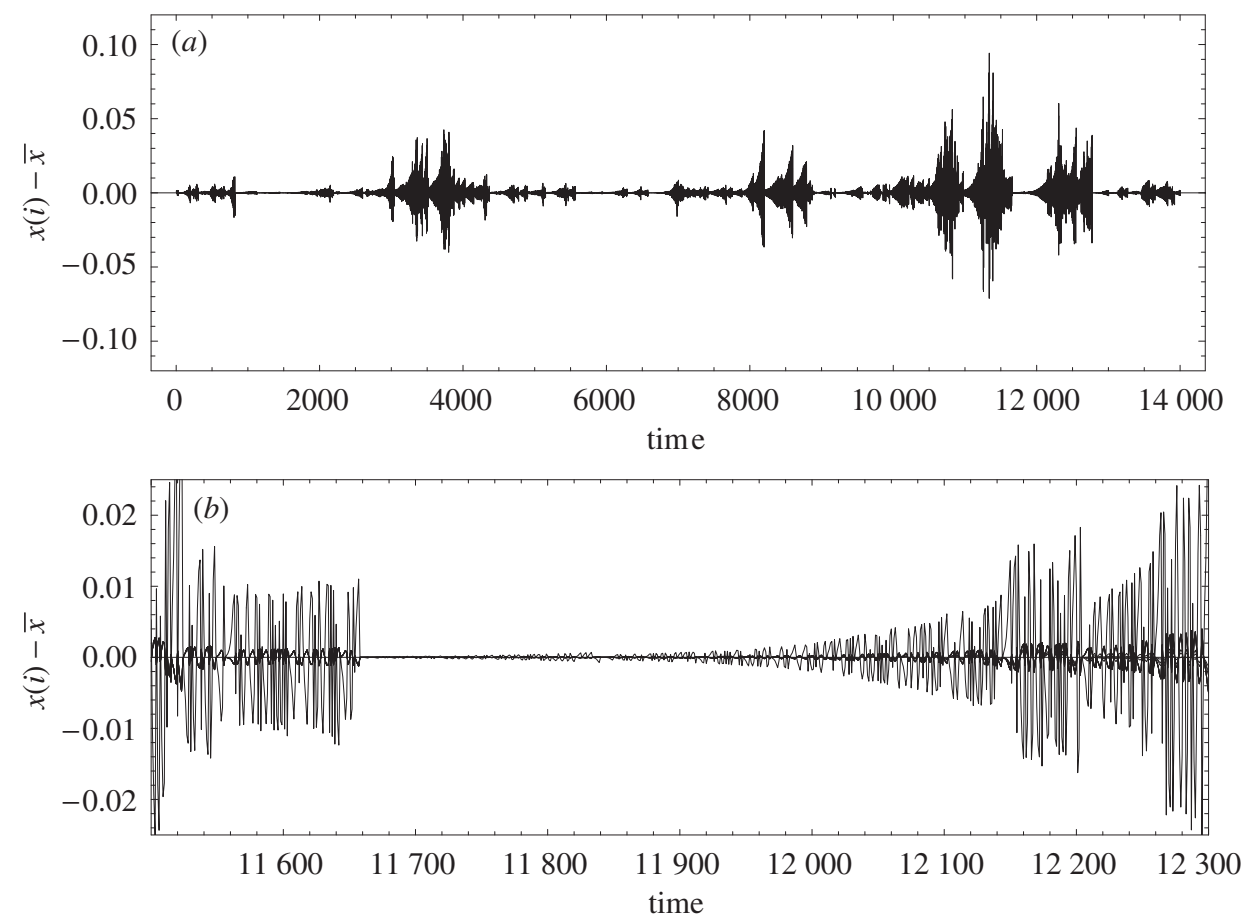

Figure 3. Unexpected local stable synchronized states in a metepopulation of 10 populations globally coupled. (a) Example of on-off intermittency: in the 'coherence impossibility region' $\left(r=4, m<m_{\mathrm{c}}=0.45\right)$, one can observe numerous time intervals during which the metapopulation is perfectly synchronized. Only one trajectory is plotted. Parameters values used are $r=4$, $m=0.455$, and $\bar{x}$ is the average abundance of the metapopulation. (b) Enlargement of $(a)$ but the 10 values $x(i)$ are plotted. Note that even though this is in the 'coherence impossibility region', the amplitudes of the 'off' state are in any case very small and the trajectories are effectively close to the state of perfect synchrony all the time (for more than $90 \%$ of the time, the trajectories remain within $5 \%$ of the coherence regime (Earn et al. 2000)).

this behaviour may be interrupted erratically via on-off intermittency (Platt et al. 1993). These complex dynamics have been reported in coupled map lattices (Xie et al. 1995; Ding \& Yang 1997; Maistrenko et al. 1999; Jiang et al. 2000) and also in ecological examples (Ferrière \& Cazelles 1999; Upadhyay et al. 2000; Cazelles 2001a,b).

\section{THE COHERENCE IMPOSSIBILITY REGION: A CLOSE UP}

It is not difficult to show that for a large range of values of $m$ inside the 'coherence impossibility region' $\left(m<m_{\mathrm{c}}=0.45\right.$, in our example with $\left.r=4\right)$, the metapopulation displays long bouts of (often perfect) synchronization. For example, when $m=0.445$, figure 3 displays the time evolution of the difference between each population abundance $x_{i}^{t}$ and the average abundance of the metapopulation $\bar{x}$. We see that there are time intervals of ecological importance (around 100 units of time) during which $\left(x_{i}^{t}-\bar{x}\right)$ stays extremely close to zero (the 'off' state defined by perfect synchrony), but accompanied by intermittent bursts (the 'on' state). Thus, even inside the 'coherence impossibility region', it is not difficult to observe tightly synchronized trajectories for a non-trivial range of $m$ values. In our example, because the intermittent bursts are in any case relatively small (usually less than $5 \%$ of the range of $\bar{x}$ ), in practical terms the populations remain closely synchronized over the entire 14000 time-steps of the simulation. Temporally stable, perfectly synchronized dynamics inside the 'coherence impossibility region' are a typical consequence of the fact that despite its positive average, $\mu_{\perp}(t)$ has finite time fluctuations that are negative, which makes the invariant synchronous manifold locally stable and attracts the trajectory toward the 'off' state intermittently. Therefore, even in the 'coherence impossibility region', there are time intervals during which the metapopulation is perfectly synchronized and, according to conventional understanding (Earn et al. 2000), the metapopulation can be endangered and subject to global exctinction by catastrophic random events.

Although our example in figure 3 for $m=0.445$ is close to the 'coherence impossibility region' boundary $m_{\mathrm{c}}=0.45$, identical effects occur under much wider conditions. One can find on-off intermittency in the interval [0.41, 0.45], which is some $10 \%$ of the 'coherence impossibility region' for $r=4$. Figure 4 displays similar results with $m=0.42$. Furthermore, figure $4 b$ displays the distribution of the absolute difference between the 10 trajectories of the metapopulation, and shows that more than $65 \%$ of the differences are less than or equal to 0.02 , i.e. within $2 \%$ of the range of metapopulation mean $\bar{x}$, and some $85 \%$ are within $5 \%$ of the range of $\bar{x}$. The effect is also dependent on the 'coherence impossibility region' and examples of 'unexpected synchronization' may also be obtained with smaller levels of dispersal. For instance, with $r=3.6$ and global coupling one finds the 'coherence impossibility region' for $m<0.20$ (see figure $2 a$ ) in which there are similar examples of 'unexpected synchronization'.

Moreover, despite the irregular and aperiodic temporal evolution of the phases with perfect synchrony (figures 3 and $4 a$ ), their probability distribution $P(D)$ depends on their duration $D$ as $P(D) \propto D^{\beta}$, with $\beta=-3 / 2$ a 

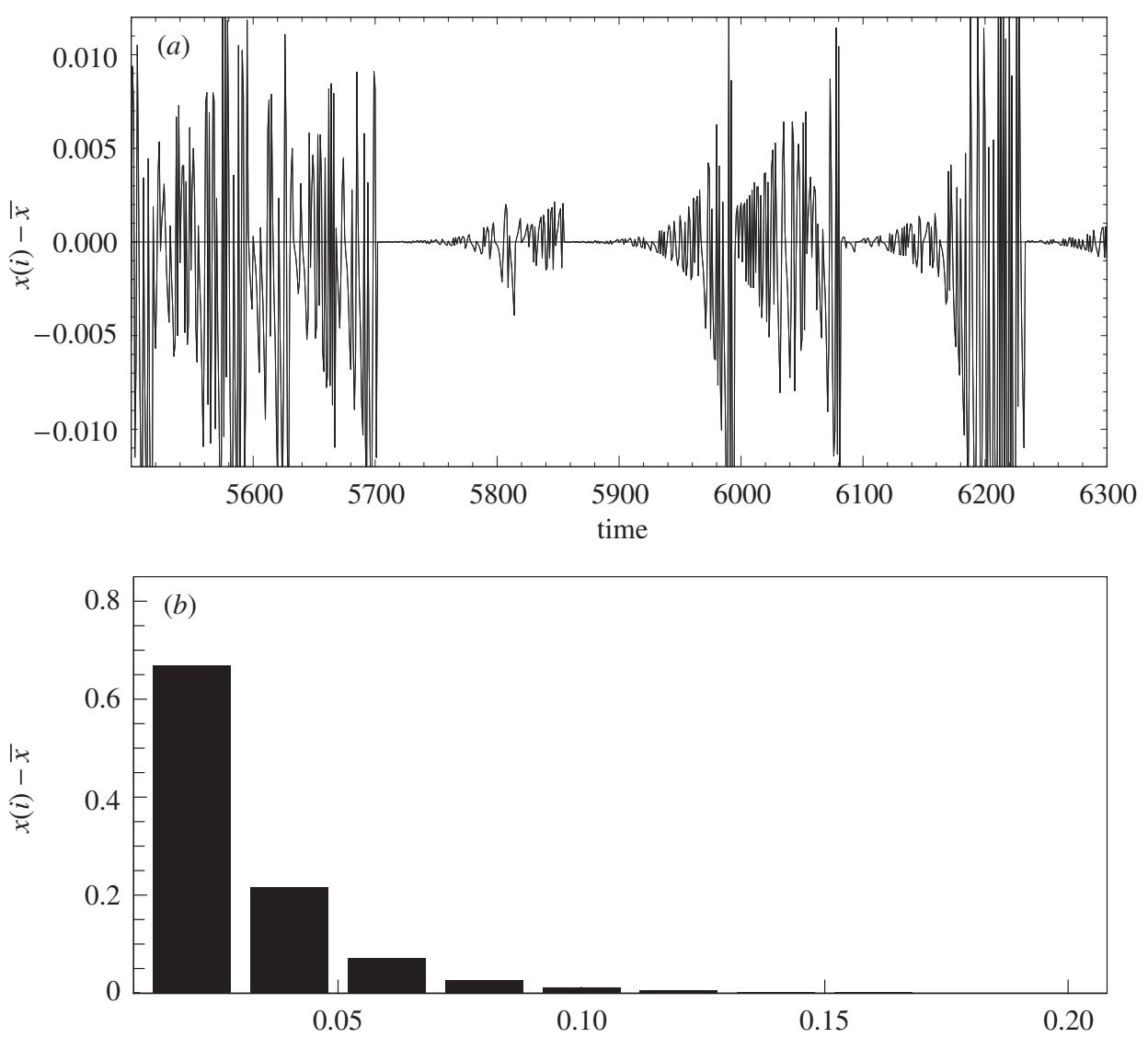

Figure 4. Unexpected local stable synchronized states in a metapopulation of 10 populations globally coupled. (a) Example of on-off intermittency. Parameters values used are $r=4, m=0.42$, and $\bar{x}$ is the average abundance of the metapopulation.

(b) Distribution of the differences between trajectories $\left(\left|x^{t}(i)-\bar{x}\right|\right)$ computed over 10000 iterations; parameters as in $(a)$.

universal scaling coefficient (Heagy et al. 1994). Figure 5 displays this probability distribution of the phases with perfect synchrony of duration $D$ and shows that this distribution follows a power law with $\beta=-3 / 2$. When entering in a 'synchronized state' the metapopulation will become highly vulnerable and this power law will provide a useful way of estimating the likelihood, in a given time interval, of a synchronized metapopulation of a given duration.

Figure 6 shows a more detailed picture of the anatomy of the 'coherence impossibility' region. In fact, it essentially breaks up into three distinct regions that may be summarized as follows.

(i) The boundary region (black and grey) for intermediate coupling strengths. This is the region where on-off intermittency of the synchronization state described above, is mostly observed.

(ii) The inner region (grey). This region consists nearly exclusively of states in which there are two clusters. Often these clusters are periodic and out-of-phase (see figure $1 a, b$ ) but they can also be chaotic with a strong out-of-phase tendency (see figure $1 c$ ). The former case is important because it identifies a synchronized periodic state in the 'coherence impossibility region', and extensive simulations reveal that the greater part of the 'inner region' is in just this state. Hence, although synchrony is often understood to be dangerous for populations, the 'coherence impossibility region' which Earn et al. (2000) argue is the least dangerous of

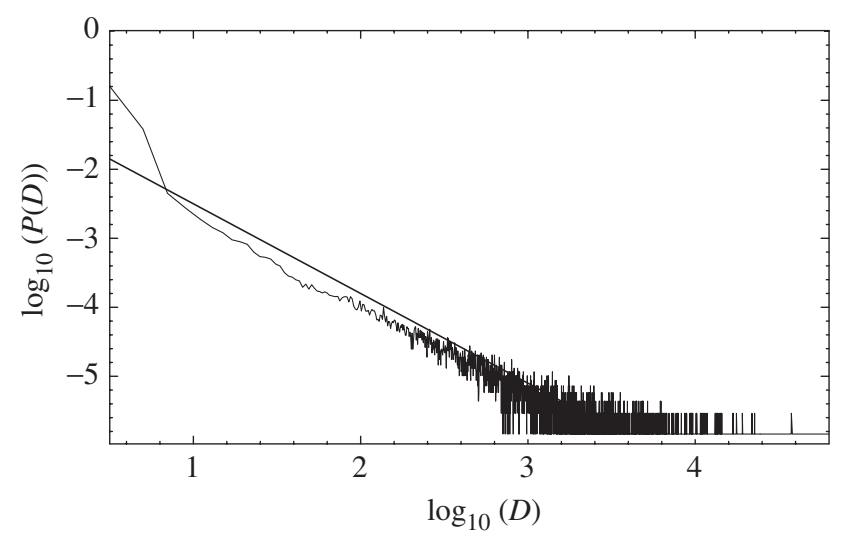

Figure 5. Probability distribution of the length of ynchronized periods $P(D)$ of duration $D$. This distribution follows the power law $P(D) \propto D^{\beta}$, with $\beta$ a universal scaling coefficient. The straight line corresponds to the theoretical distribution with slope $\beta=-3 / 2$.

all, is in fact highly synchronized. However, in this case it is predominantly out-of-phase synchronization rather than coherent oscillations.

(iii) The outer region (black) at the bottom of figure 6 . This region is truly turbulent and all populations appear to be chaotic, unsynchronized and independent. The transition to this regime manifests as plateaux in the plots of global extinction rates of Earn et al. (their fig. 3). 


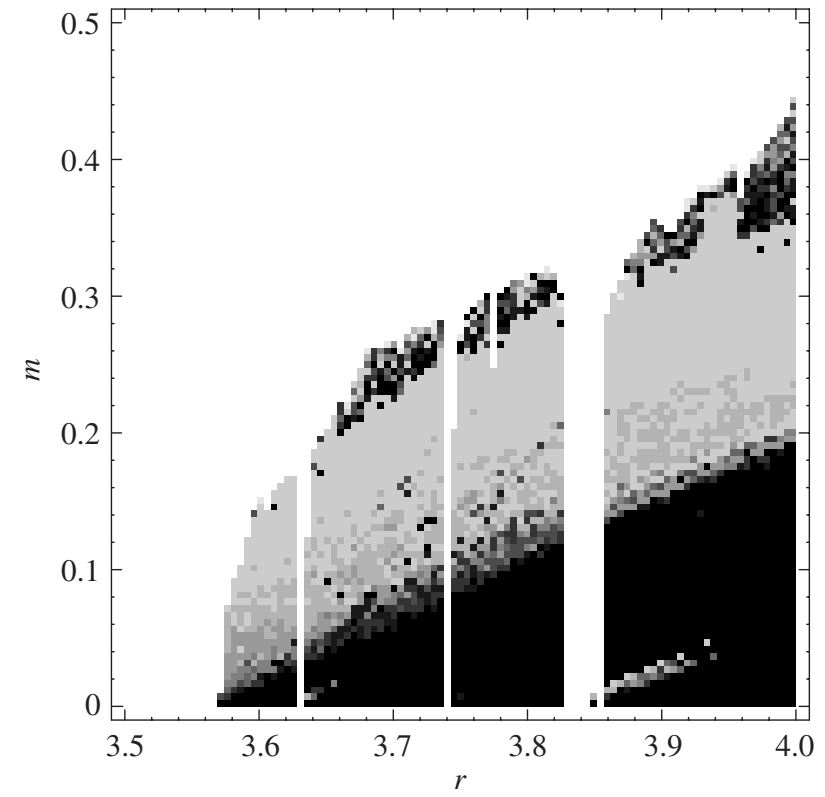

Figure 6. Complex clustering behaviour in a logistic metapopulation of $n=10$ globally coupled populations as function of the net growth rate $r$ and the dispersal coefficient $m$. The graph shows the number of clusters at the end of the simulations (10000 iterations). The grey colour is scaled from 10 clusters (black) to two clusters (light grey). Only the values in the 'coherence impossibility region' are shown.

The white colour codes for regions outside the 'coherence impossibility region'.

\section{ASYNCHRONOUS PROCESSES INDUCE SYNCHRONY}

A final point we wish to raise concerns another highly relevant ecological consideration: the asynchrony of dispersal (Huberman \& Glance 1993; Doebeli 1995; Abramson \& Zanette 1998). Taking into account the importance of environmental gradients or quasi-random timing of key environmental factors for maturation during dispersal, we have introduced random asynchrony in the dispersal phase of the model. The metapopulation was broken up into $n_{\mathrm{c}}$ disjoint 'dispersal clusters'. At each timestep a proportion of the $n$ sites was randomly assigned to the existing set of $n_{\mathrm{c}}$ 'dispersal clusters'. The local populations within each cluster were then updated, being subject to usual birth-death processes (see equation (2.2)) and to dispersal; this was carried out sequentially in turn, cluster by cluster, thereby introducing further asynchrony. This asynchrony clearly complexifies the dispersal matrix that becomes time dependent, making the computation of its subdominant eigenvalue non-trivial. But with simulations we show that this asynchrony has a pronounced effect on the stability of the spatiotemporal dynamics (Huberman \& Glance 1993; Abramson \& Zanette 1998) and can lead to perfect synchrony for some parameters that would otherwise be classed as belonging to the 'coherence impossibility region'. Figure 7 displays the evolution of the population abundances, at the end of the simulation, of each 10 subpopulations of a locally coupled metapopulation with $n_{\mathrm{c}}=5$ 'dispersal clusters' as function of $m$ and shows for $m>0.531$ a special class of perfect synchrony, namely a fixed equilibrium behaviour. This example is

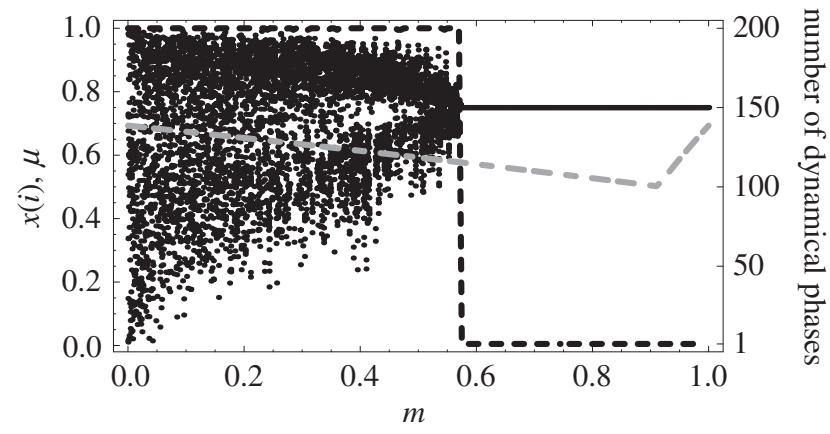

Figure 7. Bifurcation diagram with respect to dispersal coefficient $m$, showing all the values $x(i)$, for $i=1, \ldots, 10$, at the end of the simulation (black dots), in the case of a locally coupled metapopulation with asynchronous dispersal and $r=4$. The black dashed curve shows the evolution of the number of dynamical phases computed as the number of different values $\left(\left|x^{t}(i)-x^{t+1}(i)\right|>10^{-6}\right)$ for the last 200 iterations for an arbitrary given $x(i)$. For a reference, the grey dashed curve displays the evolution of transverse Lyanunov exponent $\left(\mu_{\perp}\right)$ for the case where dispersal is completely synchronous. Note that the grey dashed curve shows that $\mu_{\perp}>0$ and the dynamic without asynchrony clearly fall in the 'coherence impossibility region'. $n=10$ local populations were assigned randomly to $n_{\mathrm{c}}=5$ clusters at each iteration. Similar results were obtained with $n=10$ and $n_{\mathrm{c}}>2$.

illustrative of the unexpected effect of stochastic components on nonlinear dynamics (Vilar \& Solé 1998; Cazelles \& Boudjema 2001).

\section{CONCLUSIONS}

The synchrony of population dynamics is an issue that has become one of major concern in studies of ecological conservation. This is because coherent dynamics are perceived as dangerous in the way that they promote global extinction. Asynchronous population dynamics are understood to be more desirable because they help to 'spread the risk', thereby reducing the chance of global extinction. In a metapopulation context, it is generally accepted that metapopulation persistence is increased by increasing asynchrony in local dynamics. If the probabilities of extinction of each local population are correlated (for instance, because of dispersal or identical climatic variability), even large metapolulations may be susceptible to extinction. Then, one of the necessary conditions for metapopulation persistence is that local population dynamics are sufficiently asynchronous to make the simultaneous extinction of all local populations unlikely (e.g. Hanski 1999). There are examples of asynchronous populations; for instance, asynchrony is observed in the case of the Granville fritillary on the Âland islands (Hanski et al. 1995; Hanski 1999, chap. 11). However, there are also examples of extinctions of butterfly metapopulations in response to climatic events that have synchronous effects (Thomas et al. 1996; Sutcliffe et al. 1997).

In our analysis we have shown that patch dispersal is a powerful factor that can induce not only the usual synchronization phenomena described in the literature (e.g. Solé \& Gamarra 1998; Silva et al. 2000; Earn et al. 2000), but often surprising and hidden synchronization effects that may or may not act differently to the coherent 
oscillations described by Earn et al. (2000). The intermittent synchronization, for example, occurs in the 'coherence impossibility region', yet in contrast to the predictions of Earn et al. (2000), it is a source of dangerous coherent oscillations. The out-of-phase clusters also appear in the 'coherence impossibility region', but tend to reduce global extinction rates by generating a 'rescue effect', even though they are synchronized, i.e. again in contrast to the predictions of Earn et al. (1998). When one cluster of local populations is depressed, the other (better off) cluster acts as a source of needed immigrants.

In summary, one might keep in mind that even with simple nonlinear metapopulation models, one can observe unexpected synchronization dynamics. Apparent stochastic behaviours such as intermittent synchronization may have purely deterministic origins (figures 3 or 4). However, apparent simple deterministic dynamics and synchronized relationships may arise despite the role of important stochastic and asynchronous life-history events (figure 7).

L.S. gratefully acknowledges the support of the James S. McDonnell Foundation.

\section{REFERENCES}

Abramson, G. \& Zanette, D. H. 1998 Globally coupled maps with asynchronous updating. Phys. Rev. E 58, 4454-4460.

Alexander, J. G., Yorke, J. A., You, Z. \& Kan, I. 1992 Riddled basins. Int. 7. Bifurcation Chaos 2, 795-813.

Allen, J. C., Schaffer, W. M. \& Rosco, D. 1993 Chaos reduces species extinction by amplifying local population noise. Nature 364, 229-232.

Andreyev, Y. V. \& Dmitriev, A. S. 1999 Conditions for global synchronization in lattices of chaotic elements with local connections. Int. F. Bifurcation Chaos 9, 2165-2172.

Ashwin, P., Buescu, J. \& Stewart, I. 1994 Bubbling of attractors and synchronization of chaotic oscillators. Phys. Lett. A 193, 126-139.

Ashwin, P., Buescu, J. \& Stewart, I. 1996 From attractor to chaotic saddle: a tale of transverse instability. Nonlinearity $\mathbf{9}$, 703-737.

Balmforth, N. J., Jacobson, A. \& Provenzale, A. 1999 Synchronized family dynamics in globally coupled maps. Chaos 9, 738-754.

Blasius, B. \& Stone, L. 2000 Chaos and phase synchronization in ecological systems. Int. F. Bifurcation Chaos 10, 2361-2380.

Blasius, B., Huppert, A. \& Stone, L. 1999 Complex dynamics and phase synchronization in spatially extended ecological systems. Nature 399, 354-359.

Cazelles, B. 2001a Dynamics with riddled basins of attraction in models of interacting populations. Chaos, Solitons Fractals 12, 301-311.

Cazelles, B. $2001 b$ Blowout bifurcation with non-normal parameters in population dynamics. Phys. Rev. E 64032901.

Cazelles, B. \& Boudjema, G. 2001 The Moran effect and phase synchronization in complex spatial community dynamics. $\mathrm{Am}$. Nat. 157, 670-676.

den Boer, P. J. 1981 On the survival of populations in a heterogeneous and variable environment. Oecologia 50, 39-53.

Ding, M. \& Yang, W. 1997 Stability of synchronous chaos and on-off intermittency in coupled map lattices. Phys. Rev. E 56, 4009-4016.
Dmitriev, A. S., Shirokov, M. \& Starkov, S. O. 1997 Chaotic synchronization in ensembles of coupled maps. Inst. Electrical Electronics Engng Trans. Circuits Syst. I 44, 918-926.

Doebeli, M. 1995 Dispersal and dynamics. Theor. Popul. Biol. 47, 82-106.

Earn, D. J. D., Rohani, P. \& Grenfell, B. T. 1998 Spatial dynamics and persistence in ecology and epidemiology. Proc. R. Soc. Lond. B 265, 7-10. (DOI 10.1098/rspb.1998.0256.)

Earn, D. J. D., Levin, S. A. \& Rohani, P. 2000 Coherence and conservation. Science 290, 1360-1364.

Ferrière, R. \& Cazelles, B. 1999 Universal power laws governing chronical rarity in models of competitive communities. Ecology 80, 1505-1521.

Gade, P. M. 1996 Synchronization of oscillators with random nonlocal connectivity. Phys. Rev. E 54, 64-70.

Gade, P. M. \& Hu, C. K. 1999 Synchronization and coherence in thermodynamic coupled map lattices with intermediaterange coupling. Phys. Rev. E 60, 4966-4969.

Gotelli, 2001 A primer of ecology, 3rd edn. Sunderland, MA: Sinauer.

Hanski, I. 1999 Metapopulation ecology. Oxford University Press.

Hanski, I., Pakkala, T., Kuussaari, M. \& Lei, G. 1995 Metapopulation persistence of an endangered butterfly in a fragmented landscape. Oikos 72, 21-28.

Hassel, M. P., Comins, H. N. \& May, R. M. 1994. Species coexistence and self-organizing spatial dynamics. Nature $\mathbf{3 7 0}$, 290-292.

Heagy, J. F., Platt, N. \& Hammel, S. M. 1994 Characterization of on-off intermittency. Phys. Rev. E 49, 1140-1150.

Heino, M., Kaitala, V., Ranta, E. \& Lindström, J. 1997 Synchronous dynamics and rates of extinction in spatially structured populations. Proc. R. Soc. Lond. B 264, 481-486. (DOI 10.1098/rspb.1997.0069.)

Huberman, B. A. \& Glance, N. S. 1993 Evolutionary games and computer simulations. Proc. Natl Acad. Sci. USA 90, 7716-7718.

Jiang, Y. 2000 Globally coupled maps with time delay interactions. Phys. Lett. A 267, 342-349.

Kaneko, K. 1990 Clustering, coding switching, hierarchical ordering, and control in a network of chaotic elements. Physica D 41, 137-172.

Kozma, R. 1998 Intermediate-range coupling generates lowdimensional attractors deeply in the chaotic region of onedimensional lattices. Phys. Lett. A 244, 85-91.

Lai, Y. C., Grebogi, C., Yorke, J. A. \& Venkataramani, S. C. 1996 Riddling bifurcation in chaotic dynamical systems. Phys. Rev. Lett. 77, 55-58.

Maistrenko, Y. L., Maistrenko, V. L., Popovych, O. \& Mosekilde, E. 1999 Desynchronization of chaos in coupled logistic maps. Phys. Rev. E 60, 2817-2830.

Maistrenko, Y. L., Popovych, O. \& Hasler, M. 2000 On strong and weak chaotic partial synchronization. Int. F. Bifurcation Chaos 10, 179-203.

Platt, N., Spiegel, E. A. \& Tresser, C. 1993 On-off intermittency: a mechanism for bursting. Phys. Rev. Lett. 70, 279282.

Rosenblum, M. G., Pikovsky, A. S. \& Kurths, J. 1996 Phase synchronization of chaotic oscillators. Phys. Rev. Lett. 76, 18041807.

Rosenblum, M. G., Pikovsky, A. S. \& Kurths, J. 1997 From phase synchronization to lag synchronization in coupled chaotic oscillators. Phys. Rev. Lett. 78, 4193-4196.

Rulkov, N. F., Suschik, M. M., Tsimring, L. S. \& Abarbanel, H. D. I. 1995 Generalized synchronization of chaos in directionally coupled chaotic systems. Phys. Rev. E 51, 980994.

Silva, J. A. L.., De Castro, M. L. \& Justo, A. R. 2000 Synchronism in metapopulation model. Bull. Math. Biol. 62, 337-349. 
Solé, R. V. \& Gamarra, J. G. P. 1998 Chaos, dispersal and extinction in coupled ecosysytem. F. Theor. Biol. 193, 539541.

Sutcliffe, O. L., Thomas, C. D., Yates, T. J. \& Greatorex-Davis, J. N. 1997 Correlated extinctions, colonisations and population fluctuations in a highly connected ringlet butterfly metapopulation. Oecologia 109, 235-241.

Thomas, G. D., Singer, M. C. \& Boughton, D. A. 1996 Catastrophic extinction of population sources in butterfly metapopulation Am. Nat. 148, 957-975.
Upadhyay, R. K., Iyengar, S. R. K. \& Rai, V. 2000 Stability and complexity in ecological systems. Chaos, Solitons Fractals 11, 533-542.

Vilar, J. M. G. \& Solé, R. V. 1998 Effects of noise in symmetric two-species competition. Phys. Rev. Lett. 80, 4099-4102.

Xie, F., Hu, G. \& Qu, Z. 1995 On-off intermittency in a coupled-map lattice system. Phys. Rev. E 52, 1265-1268.

As this paper exceeds the maximum length normally permitted, the authors have agreed to contribute to production costs. 